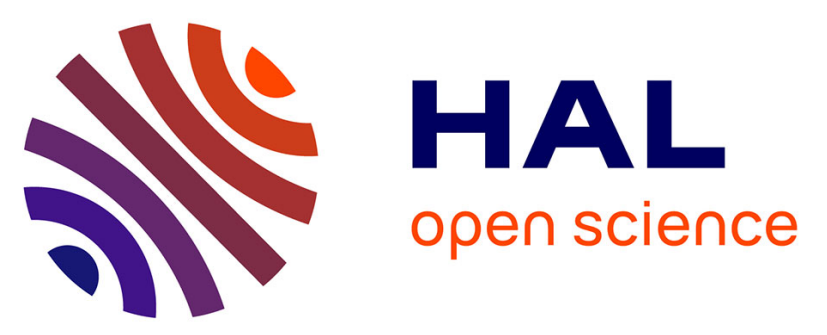

\title{
Homogeneous Reforming of Aqueous Ethylene Glycol to Glycolic Acid and Pure Hydrogen Catalyzed by Pincer-Ruthenium Complexes Capable of Metal-Ligand Cooperation
}

You-Quan Zou, Niklas von Wolff, Michael Rauch, Moran Feller, Quan-Quan Zhou, Aviel Anaby, Yael Diskin-Posner, Linda J W Shimon, Liat Avram, Yehoshoa Ben-David, et al.

\section{- To cite this version:}

You-Quan Zou, Niklas von Wolff, Michael Rauch, Moran Feller, Quan-Quan Zhou, et al.. Homogeneous Reforming of Aqueous Ethylene Glycol to Glycolic Acid and Pure Hydrogen Catalyzed by Pincer-Ruthenium Complexes Capable of Metal-Ligand Cooperation. Chemistry - A European Journal, 2020, 10.1002/chem.202005450 . hal-03090552

\section{HAL Id: hal-03090552 \\ https://hal.science/hal-03090552}

Submitted on 29 Dec 2020

HAL is a multi-disciplinary open access archive for the deposit and dissemination of scientific research documents, whether they are published or not. The documents may come from teaching and research institutions in France or abroad, or from public or private research centers.
L'archive ouverte pluridisciplinaire HAL, est destinée au dépôt et à la diffusion de documents scientifiques de niveau recherche, publiés ou non, émanant des établissements d'enseignement et de recherche français ou étrangers, des laboratoires publics ou privés. 


\title{
Homogeneous Reforming of Aqueous Ethylene Glycol to Glycolic Acid and Pure Hydrogen Catalyzed by Pincer-Ruthenium Complexes Capable of Metal-Ligand Cooperation
}

\author{
You-Quan Zou, ${ }^{[a], \dagger}$ Niklas von Wolff, ${ }^{[a],[b], \dagger}$ Michael Rauch, ${ }^{[a]}$ Moran Feller, ${ }^{[a]}$ Quan-Quan, Zhou, ${ }^{[a]}$ Aviel \\ Anaby, ${ }^{[\mathrm{a}]}$ Yael Diskin-Posner, ${ }^{[\mathrm{c}]}$ Linda J. W. Shimon, ${ }^{[\mathrm{c}]}$ Liat Avram, ${ }^{[\mathrm{cl}}$ Yehoshoa Ben-David, ${ }^{[\mathrm{a}]}$ and David \\ Milstein*[a]
}

\begin{abstract}
Glycolic acid is a useful and important $\alpha$-hydroxy acid that has broad applications. Herein, we report the homogeneous ruthenium catalyzed reforming of aqueous ethylene glycol to generate glycolic acid as well as pure hydrogen gas, without concomitant $\mathrm{CO}_{2}$ emission. This approach provides a clean and sustainable direction to glycolic acid and hydrogen, based on inexpensive, readily available and renewable ethylene glycol using $0.5 \mathrm{~mol} \%$ of catalyst. In-depth mechanistic experimental and computational studies highlight key aspects of the $\mathrm{PNNH}$-ligand framework involved in this transformation.
\end{abstract}

\section{Introduction}

The development of green and atom efficient protocols in organic synthesis is an important goal. ${ }^{[1]}$ Indeed, over the last decade, progress has been made in the design and discovery of highly atom efficient transformations in organic redox chemistry. In this respect, transition metal complexes that are able to activate strong bonds via metal-ligand cooperation (MLC) are particularly fruitful in catalysis, ${ }^{[2]}$ enabling the dehydrogenation of readily available starting materials, such as alcohols and amines under mild conditions and very high atom efficiency in the synthesis of useful amides and esters via acceptorless dehydrogenative coupling (ADC) reactions. Additionally, these complexes have been utilized to hydrogenate a variety of oxidized substrates, such as amides, esters, carbonates, carbamates and ureas to fine and useful chemicals. ${ }^{[3]}$ Given the great potential of ADC catalysts in greener synthetic routes, it is desirable to extend the possible field of application to key molecular targets. In addition, gaining a more thorough understanding of their reactivity and bond activation patterns may help the design of new reaction schemes in the future to access desirable molecules in an efficient manner.

[a] Dr. Y.-Q. Zou, ${ }^{[\dagger]}$ Dr. N. von Wolff, ${ }^{[+]}$Dr. M. Rauch, Dr. M. Feller. Dr. Q.-Q. Zhou, Dr. A. Anaby, Y. Ben-David, Prof. Dr. D. Milstein

Department of Organic Chemistry

Weizmann Institute of Science

Rehovot 76100, Israel

E-mail: david.milstein@weizmann.ac.il

[†] These authors contributed equally to this work

[b] Present address: Université de Paris, Laboratoire d'Electrochimie Moléculaire, CNRS, F-75006 Paris, France

[c] Dr. Y. Diskin-Posner, Dr. L. J. W. Shimon, Dr. L. Avram Department of Chemical Research Support

Weizmann Institute of Science

Rehovot 76100 , Israel

Supporting information for this article is given via a link at the end of the document
Being used today in a variety of fields, ${ }^{[4]}$ ranging from skin care, adhesives and polymers to the textile industry, glycolic acid is an essential chemical commodity with a forecasted US market share of over 400 million USD by $2024 .{ }^{[5]}$ Nevertheless, the current protocols for its synthesis rely on high pressure formaldehyde carbonylation using $\mathrm{CO}$ at high temperatures, ${ }^{[6]}$ or hydrolysis of chloroacetic acid or methyl 2-hydroxyacetate $;^{[7,8]}$ processes that are associated with the generation of stoichiometric amounts of waste, multiple synthetic steps, and the use of toxic reagents. The environmentally benign, direct generation of glycolic acid from readily accessible starting materials under mild and atom efficient conditions is highly desirable.

Given the outstanding performance of pincer-type complexes in the ADC reactions of alcohols, ${ }^{[3]}$ we are interested in understanding if and how pincer complexes might enable the generation of glycolic acid from readily available alcohols. In this respect, ethylene glycol ${ }^{[9]}$ as a feedstock is of particular interest, as it is potentially biomass-derived and already used on an industrial scale. ${ }^{[10]}$ Pioneering work by the Dumesic group elegantly demonstrated that aqueous ethylene glycol can be reformed to hydrogen, carbon dioxide and short alkanes at elevated temperatures using $\mathrm{Pt}_{/} / \mathrm{Al}_{2} \mathrm{O}_{3}{ }^{[11]}$ and Raney-NiSn ${ }^{[12]}$ catalysts at $225^{\circ} \mathrm{C}$ or $265^{\circ} \mathrm{C}$. Despite the great interest in the use of atom efficient reforming of ethylene glycol, only a single heterogenous system developed by Bitter and co-workers has been described to catalyze the transformation of ethylene glycol to glycolic acid and hydrogen. At 150 or $180{ }^{\circ} \mathrm{C}$ using carbon nanofiber (CNF)- supported copper and nickel nanoparticles, glycolic acid could be obtained from ethylene glycol, ${ }^{[13]}$ whereas a Ni/CNF catalyst led to further decomposition to formic acid, hydrogen and carbon dioxide (Scheme 1a).

Homogeneous reforming of ethylene glycol to generate hydrogen was reported by the groups of Cole-Hamilton ${ }^{[14]}$ and Beller, ${ }^{[15 a]}$ although the generation of glycolic acid was not confirmed. The group of Grützmacher reported the oxidative dehydrogenation of alcohols and polyalcohols, including ethylene glycol, to carboxylic acids using a homogeneous rhodium catalyst under mild conditions, albeit in the presence of sacrificial hydrogen acceptors such as ketones or methyl methacrylate. ${ }^{[16]}$ Reforming of ethylene glycol to glycolic acid homogeneously catalyzed by an iridium complex (4 mol\%) was reported recently, although the catalytic mechanism was not studied. ${ }^{[17]}$ Very recently, homogeneous iridium-catalyzed dehydrogenative crosscoupling of ethylene glycol and methanol to lactic acid was also disclosed by Tu, Xu and co-workers. ${ }^{[18]}$ The scarcity of reports on catalytic reforming of aqueous ethylene glycol to glycolic acid, despite its significant promise, demonstrates the need for new catalytic systems as well as a deeper understanding of the reaction mechanism. Herein, we report a fully characterized 
homogeneously catalyzed reforming of aqueous ethylene glycol to glycolic acid and exclusively hydrogen gas at low catalyst loading ( $0.1 \mathrm{~mol} \%$ on large scale). Mechanistic studies, supported by synthesis of potential intermediates, X-ray crystallography, nuclear magnetic resonance (NMR) experiments and density functional theory (DFT) calculations are presented to highlight the key steps of this transformation.

(a) Heterogeneously catalyzed reforming aqueous ethylene glycol

$$
\begin{aligned}
& \text { Dumesic's work: } \quad \mathrm{Pt} / \mathrm{Al}_{2} \mathrm{O}_{3} \\
& \mathrm{HO}{ }_{\mathrm{OH}}+\mathrm{H}_{2} \mathrm{O} \underset{225 \text { or } 265^{\circ} \mathrm{C}}{\stackrel{\text { or Raney-NiSn }}{\longrightarrow}} \mathrm{H}_{2}+\mathrm{CO}_{2}+\mathrm{CH}_{4}+\mathrm{C}_{2} \mathrm{H}_{6}
\end{aligned}
$$

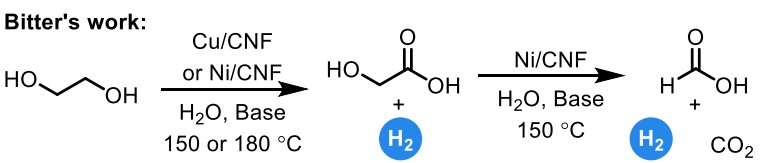

(b) Our previous work: liquid organic hydrogen carrier based on ethylene glycol

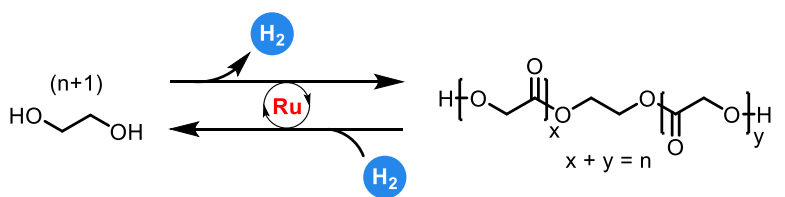

(c) This work: reforming aqueous ethylene glycol to glycolic acid and hydrogen

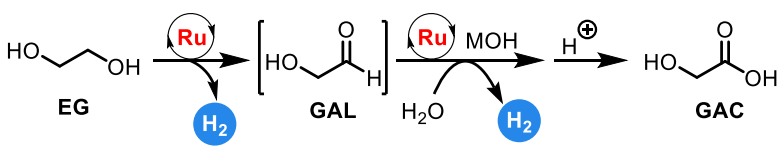

-highly efficient whigh purity of $\mathrm{H}_{2} \backsim$ no $\mathrm{CO}_{2}$ emission $\square$ mechanism usustainable synthesis of glycolic acid $\quad$ mild reaction conditions

Scheme 1. (a) Representative examples of heterogeneously catalyzed reforming of aqueous ethylene glycol. (b) Ethylene glycol as a reversible hydrogen carrier. (c) Homogeneously catalyzed reforming of aqueous ethylene glycol to glycolic acid and $\mathrm{H}_{2}$.

\section{Results and Discussion}

During recent years, our group has reported several acceptorless dehydrogenative coupling reactions of alcohols with alcohols, ${ }^{[19]}$ amines ${ }^{[20]}$ and water ${ }^{[21]}$ to the corresponding esters, amides and acids. Very recently, we described a liquid organic hydrogen carrier system based on ethylene glycol, which enabled the efficient and reversible loading and discharge of hydrogen using a single ruthenium pincer complex (Scheme 1b). ${ }^{[22]}$ Based on this report, we envisioned that ethylene glycol would undergo dehydrogenation to glycolaldehyde (GAL) in the presence of a ruthenium pincer complex via MLC, accompanied by hydrogen release (Scheme 1c). Subsequently, the interception of glycolaldehyde by water and base could liberate a second molecule of hydrogen and enable access to glycolic acid (GAC) after acid workup.

To examine the feasibility of the above-proposed reforming process, we evaluated the acridine-type PNP complexes, Ru-1 and Ru-2, that gave excellent results in the dehydrogenative coupling of ethylene glycol to oligoesters (Table 1). ${ }^{[22]}$ The reforming of aqueous ethylene glycol was attempted using 0.5 mol\% catalyst loading and 5 equivalents of potassium hydroxide at $115^{\circ} \mathrm{C}$ (oil bath temperature) in a $1: 1$ mixture of water $(1.0 \mathrm{~mL}$ ) and THF $(1.0 \mathrm{~mL}) .^{[23]}$ Somewhat surprisingly, these catalysts showed only sluggish reactivity (Table 1 , entries 1 and 4 ) giving a maximum of $14 \mathrm{~mL}$ of hydrogen. Increasing the temperature to $150{ }^{\circ} \mathrm{C}$ resulted in increased volumes of $\mathrm{H}_{2}$ up to $71 \mathrm{~mL}$ (entries 3 and 6). On the other hand, the PNN and PNP ruthenium complexes Ru-3 to Ru-7, developed by our group, gave more promising results (entries 7-11). Complex Ru-4 performed best, delivering $195 \mathrm{~mL}$ of hydrogen gas (81\% yield, theoretical volume of hydrogen: $240 \mathrm{~mL}$ ) after 48 hours at $115^{\circ} \mathrm{C}$ (entry 8 ). Gas chromatographic (GC) analysis of the gas phase indicated that the evolved hydrogen was pure (Figure S28). NMR analysis of the crude reaction mixture indicated that only potassium 2hydroxyacetate was formed (see Figures $\mathrm{S} 12-13$ for details). After workup with $6 \mathrm{M} \mathrm{HCl}$, glycolic acid was formed in $78 \%$ yield (entry 8, Figures S20-21).

Table 1. Optimization of the reaction conditions using PNN and PNP pincer complexes Ru-1 to Ru-7

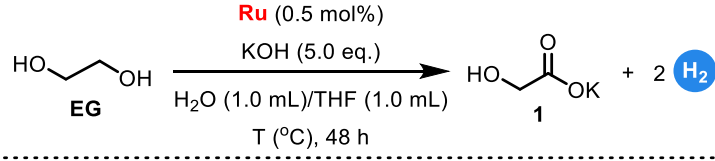

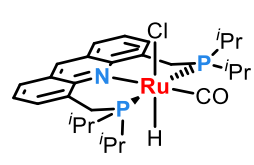

Ru-1
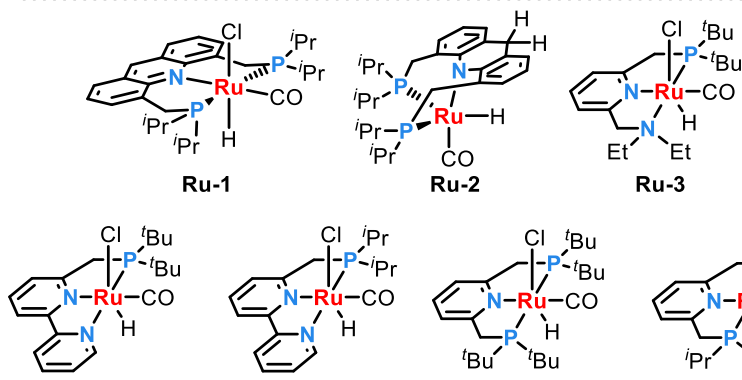

Ru-4

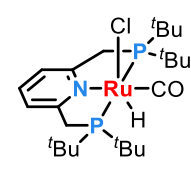

Ru-6

\begin{tabular}{|c|c|c|c|c|}
\hline Entry ${ }^{[a]}$ & Ru & $\mathrm{T}\left({ }^{\circ} \mathrm{C}\right)$ & $\mathrm{V}\left(\mathrm{H}_{2}, \mathrm{~mL}\right)^{[\mathrm{b}]}$ & Yield $(1, \%)^{[c]}$ \\
\hline 1 & Ru-1 & 115 & 14 & 6 \\
\hline 2 & Ru-1 & 135 & 22 & 9 \\
\hline 3 & Ru-1 & 150 & 71 & 30 \\
\hline 4 & Ru-2 & 115 & $<1$ & N.D. \\
\hline 5 & Ru-2 & 135 & 68 & 28 \\
\hline 6 & Ru-2 & 150 & 71 & 30 \\
\hline 7 & Ru-3 & 115 & 181 & 75 \\
\hline 8 & Ru-4 & 115 & 195 & $81(78)^{[\mathrm{d}]}$ \\
\hline 9 & Ru-5 & 115 & 146 & 61 \\
\hline 10 & Ru-6 & 115 & 113 & 47 \\
\hline 11 & Ru-7 & 115 & 170 & 71 \\
\hline
\end{tabular}

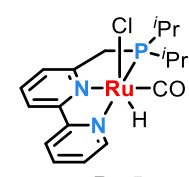

Ru-5

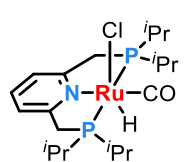

Ru-7 [a] Reaction conditions: ethylene glycol $(5.0 \mathrm{mmol})$, Ru cat. $(0.5 \mathrm{~mol} \%), \mathrm{KOH}$ (25 mmol), $\mathrm{H}_{2} \mathrm{O}(1.0 \mathrm{~mL})$ and $\mathrm{THF}(1.0 \mathrm{~mL})$ at $115^{\circ} \mathrm{C}$ (bath temperature) for 48 hours. [b] Hydrogen gas was collected every 24 hours. [c] Yields are based on the volume of collected hydrogen gas. [d] Yield of glycolic acid is shown in 
parentheses, as determined by ${ }^{1} \mathrm{H}$ NMR of the crude reaction mixture after acid workup using acetonitrile as an internal standard. N.D.: not determined.

To our delight, upon screening PNNH-based complexes (Table 2) we found that using Ru-8 the yield of potassium 2hydroxyacetate was increased to $89 \%$ and $214 \mathrm{~mL}$ of pure hydrogen (Figure S29) were collected after 48 hours (Table 2, entry 2). At the same time, glycolic acid was formed in $88 \%$ yield after acid workup of the crude reaction mixture (Figures S22-23) indicating an excellent correlation between the yield of glycolic acid and the amount of hydrogen evolved. Additionally, Ru-9 and Ru-10 also catalyzed the reaction, albeit in moderate yields of hydrogen and potassium 2-hydroxyacetate (entries 3 and 4). Notably, doubling the water loading liberated $223 \mathrm{~mL}$ of pure hydrogen (Figure S30) and further increased the yield of glycolic acid to $91 \%$ (entry 5, Figures S24-25). The glycolic acid was isolated in $84 \%$ yield after removing the water, followed by extraction with THF. Lowering the reaction temperature to $100{ }^{\circ} \mathrm{C}$ led to decreased reaction efficiency (entry 6). Using $\mathrm{NaOH}$ instead of $\mathrm{KOH}$ provided comparable results, yielding $94 \%$ of hydrogen and $93 \%$ of glycolic acid after workup (entry 7 , Figures S26-27). Lower loadings of $\mathrm{NaOH}$ resulted in formation of less hydrogen gas (193 $\mathrm{mL}$, entry 8$)$. Using water as the only solvent (single phase) resulted in a sluggish reaction (entry 9), but it proceeded well at higher temperatures, generating $48 \%$ and $70 \%$ yields of potassium 2-hydroxyacetate at $135^{\circ} \mathrm{C}$ and $150{ }^{\circ} \mathrm{C}$, respectively (entries 10 and 11 ).

Table 2. Production of glycolic acid and hydrogen from aqueous ethylene glycol catalyzed by PNNH complexes Ru-8, 9, 10

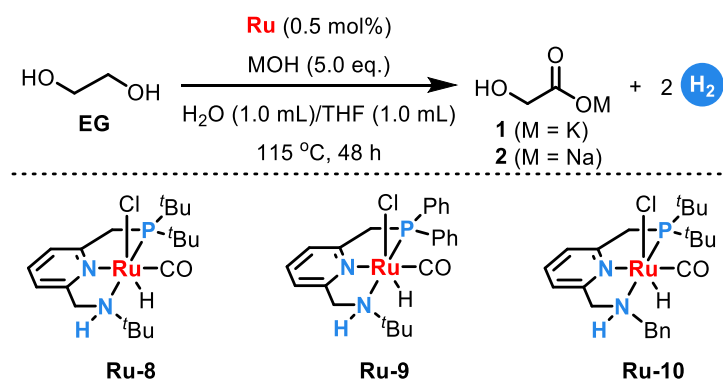

\begin{tabular}{|c|c|c|c|c|}
\hline Entry ${ }^{[a]}$ & $\mathbf{R u}$ & $\mathrm{MOH}$ & $\mathrm{V}\left(\mathrm{H}_{2}, \mathrm{~mL}\right)^{[\mathrm{b}]}$ & Yield $(1, \%)^{[c]}$ \\
\hline 1 & Ru-4 & $\mathrm{KOH}$ & 195 & $81(78)^{[d]}$ \\
\hline 2 & Ru-8 & $\mathrm{KOH}$ & 214 & $89(88)^{[d]}$ \\
\hline 3 & Ru-9 & $\mathrm{KOH}$ & 138 & 58 \\
\hline 4 & Ru-10 & $\mathrm{KOH}$ & 166 & 69 \\
\hline $5^{[e]}$ & Ru-8 & $\mathrm{KOH}$ & 223 & $93(91)^{[d]}$ \\
\hline $6^{[f]}$ & Ru-8 & $\mathrm{KOH}$ & 79 & 33 \\
\hline $7^{[\mathrm{e}]}$ & Ru-8 & $\mathrm{NaOH}$ & 226 & $94(93)^{[d]}$ \\
\hline $8^{[e, g]}$ & Ru-8 & $\mathrm{NaOH}$ & 193 & 80 \\
\hline $9^{[\mathrm{h}]}$ & Ru-8 & $\mathrm{KOH}$ & 29 & 12 \\
\hline $10^{[h, i]}$ & Ru-8 & $\mathrm{KOH}$ & 116 & 48 \\
\hline
\end{tabular}

Ru-8

$\mathrm{KOH}$

167

70

[a] Reaction conditions: ethylene glycol $(5.0 \mathrm{mmol})$, Ru cat. $(0.5 \mathrm{~mol} \%), \mathrm{MOH}$ (25 mmol, $\mathrm{M}=\mathrm{K}, \mathrm{Na}), \mathrm{H}_{2} \mathrm{O}(1.0 \mathrm{~mL})$ and THF $(1.0 \mathrm{~mL})$ at $115{ }^{\circ} \mathrm{C}$ (bath temperature) for 48 hours. [b] Hydrogen was collected every 24 hours. [c] Yields are based on the volume of collected hydrogen gas. [d] Yield of glycolic acid is shown in parentheses, as determined by ${ }^{1} \mathrm{H}$ NMR of the crude reaction mixture after acid workup using acetonitrile as an internal standard. [e] $\mathrm{H}_{2} \mathrm{O}(2.0 \mathrm{~mL})$ was used. [f] Reaction was performed at $100{ }^{\circ} \mathrm{C}$. [g] $\mathrm{NaOH}(15 \mathrm{mmol})$ was used. [h] $\mathrm{H}_{2} \mathrm{O}(3.0 \mathrm{~mL})$ was used as the reactant and solvent without THF. [i] Reaction was performed at $135^{\circ} \mathrm{C}$. [j] Reaction was performed at $150{ }^{\circ} \mathrm{C}$. Grey rows are indicative of optimal conditions.

After establishing the optimized reaction conditions, we investigated the scalability of our methodology. As shown in Figure 1, using only $0.1 \mathrm{~mol} \%$ of Ru-8, $10 \mathrm{mmol}$ of ethylene glycol were efficiently dehydrogenated to form hydrogen gas and sodium 2-hydroxyacetate in a mixture of THF $(2.0 \mathrm{~mL})$ and water $(4.0 \mathrm{~mL})$. During the first 48 hours, the reaction was relatively fast, releasing $282 \mathrm{~mL}$ of hydrogen. Subsequently, the reaction slowed down, likely a result of the decreased concentration of ethylene glycol. After 120 hours, $349 \mathrm{~mL}$ pure hydrogen gas (yield of hydrogen at this stage: $73 \%$ ) was collected (red curve, see Figure $\mathrm{S} 4$ for details). The reforming reaction also proceeded quite well on a $30 \mathrm{mmol}$ scale, delivering $921 \mathrm{~mL}$ of pure hydrogen gas (yield of hydrogen at this stage: $64 \%$ ) after 120 hours (blue curve, $3 \mathrm{~mL}$ THF and $6 \mathrm{~mL}$ water were used, see Figure S5 for details). A catalyst recycling experiment indicated that the catalytic species was still active after 72 hours, albeit at a reduced rate (Figure S6, see SI for details).
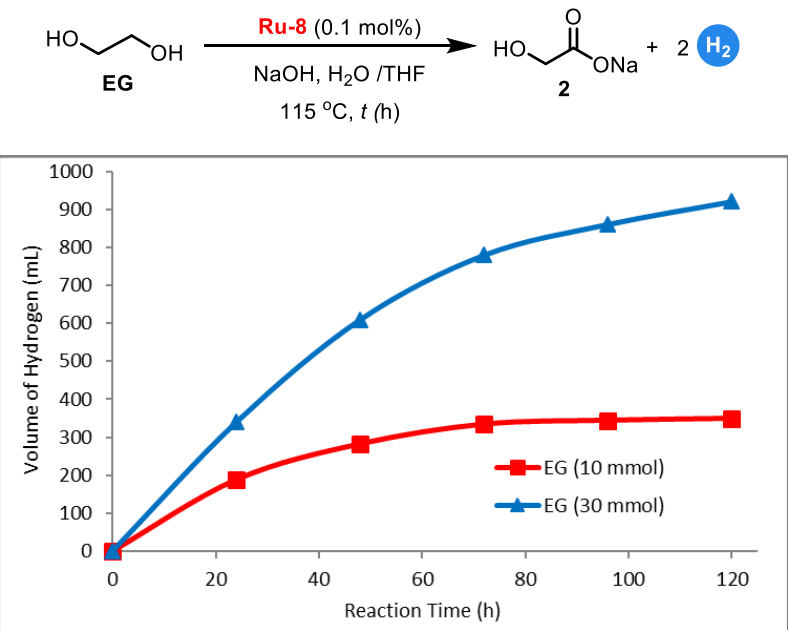

Figure 1. Reforming aqueous ethylene glycol to hydrogen and sodium 2hydroxyacetate using $0.1 \% \mathrm{~mol}$ of Ru-8.

Even though complexes Ru-1 and Ru-2 were shown to be efficient in the dehydrogenative coupling of ethylene glycol to oligoesters, ${ }^{[22]}$ the PNNH complex Ru-8 showed superior performance in the reforming reaction. In order to understand the efficiency of the PNNH-family of complexes, we sought to investigate its possible modes of bond activation. An entry point to a possible catalytic cycle is the deprotonation of Ru-8 by base. Previous experimental and theoretical reports have shown that in the presence of excess base (and absence of a protic medium), a double deprotonated anionic species was formed, in which the $\mathrm{CH}_{2}$-group of $\mathrm{P}$-arm was still present. ${ }^{[24]}$ On the other hand, using 
only 1.1 equivalents of base ${ }^{1} \mathrm{H},{ }^{31} \mathrm{P}\left\{{ }^{1} \mathrm{H}\right\},{ }^{13} \mathrm{C}\left\{{ }^{1} \mathrm{H}\right\}$ DEPTQ and ${ }^{1} \mathrm{H}\left\{{ }^{13} \mathrm{C}\right\} \mathrm{HSQC}$ NMR clearly showed that among the three acidic sites (P-arm, $\mathrm{N}$-arm and $\mathrm{NH}$-group) only $\mathrm{P}$-arm deprotonation took place (Figures S33-37). This finding is further corroborated by DFT calculations, showing that the P-arm deprotonated species 3a is 3.6 and $6.8 \mathrm{kcal}^{\mathrm{mol}}{ }^{-1}$ more stable than the $\mathrm{NH}$ deprotonated species $\mathbf{3 b}$ and the $\mathrm{N}$-arm deprotonated $\mathbf{3} \mathbf{c}$, respectively (Scheme $2 b$, see the SI for details). Furthermore, in a protic medium (such as the crude reaction mixture), no double deprotonated species is observed even in the presence of excess base. Treatment of the resulting $\mathrm{P}$-arm deprotonated mixture with 1 atmosphere of $\mathrm{H}_{2}$ at room temperature in THF converged to the aromatized trans-dihydride complex 4 (Scheme 2a). Complex 4 was only stable under an atmosphere of hydrogen, and was fully characterized under $\mathrm{H}_{2}$ (Figures $\mathrm{S} 38-42$, see $\mathrm{SI}$ for details).

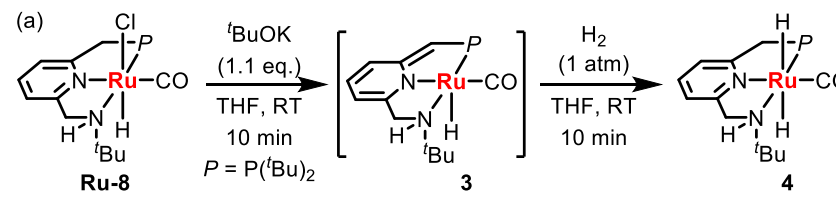

(b)
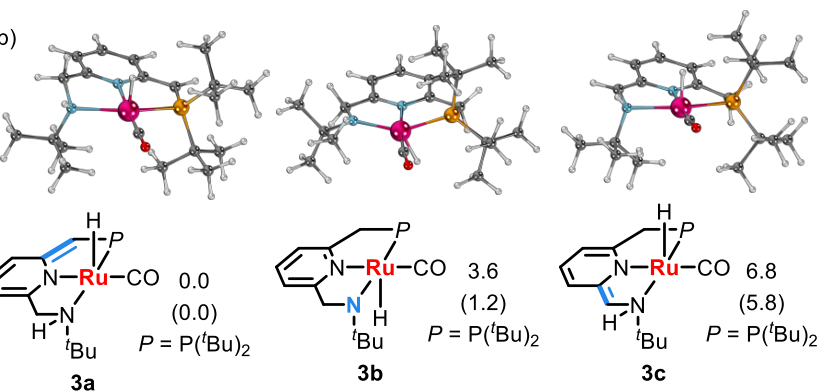

Scheme 2. (a) Deprotonation of Ru-8 followed by reaction with $\mathrm{H}_{2}$. (b) Site of deprotonation of Ru-8 by DFT calculations. Values are Gibbs free energies in kcal. $\mathrm{mol}^{-1}$ with respect to $\mathbf{3 a}$ in THF (Values in parentheses are gas phase values, 298K)

Since attempts to crystallize the product of the reaction between dearomatized intermediate 3 and ethylene glycol were unsuccessful, we used the PNN complex Ru-4 as surrogate (which exhibits good catalytic performance, see Table 1, entry 8). Deprotonating Ru-4 (using 1.2 equivalent of ${ }^{\mathrm{t}} \mathrm{BuOK}$ ) followed by reaction with ethylene glycol led to formation of the aromatized complex 6 via the dearomatized species $5,{ }^{[25]}$ as witnessed by NMR spectroscopy (Scheme 3). In the ${ }^{1} \mathrm{H}$ NMR spectrum (Figure S43), the hydride is shifted downfield to $-16.14 \mathrm{ppm}$ (doublet, ${ }^{2} \mathrm{~J}_{\mathrm{PH}}$ $=25 \mathrm{~Hz}$ ), consistent with the vacant position trans to the hydride of $\mathbf{5}$, now being occupied by the alkoxo group. A broad singlet appeared at $\delta=103.4 \mathrm{ppm}$ in the ${ }^{31} \mathrm{P}\left\{{ }^{1} \mathrm{H}\right\}$ NMR spectrum (Figure $\mathrm{S} 46)$, which is also consistent with a saturated aromatic complex. The IR spectrum showed a strong absorption band at $1907 \mathrm{~cm}^{-1}$ (Figure S47). Slow evaporation of the mixture led to formation of crystals suitable for X-ray diffraction. As shown in Figure 2, a neutral distorted octahedral ruthenium complex was formed, with the alkoxide of ethylene glycol coordinated to the metal center. In the unit cell of the crystal, $\mathrm{H}$-bonding with another molecule of ethylene glycol to the alkoxide ligand is observed (see SI for details).

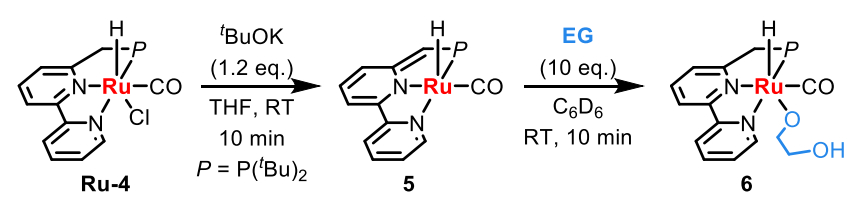

Scheme 3. Dearomatization of complex Ru-4 and reaction with ethylene glycol.

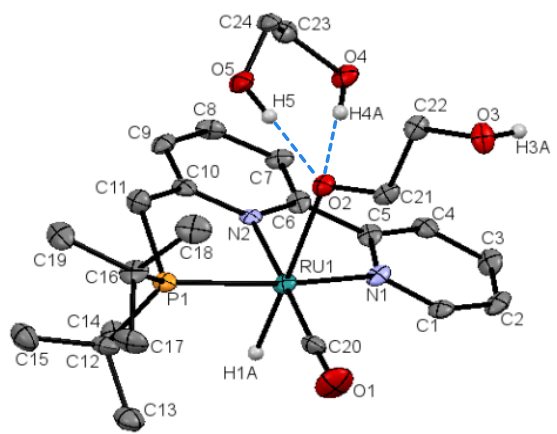

Figure 2. X-ray crystal structure of complex 6 . Atoms are presented as thermal ellipsoids at $50 \%$ probability level. Hydrogen atoms are omitted for clarity except the hydride $\mathrm{H} 1 \mathrm{~A}, \mathrm{H} 5, \mathrm{H} 3 \mathrm{~A}$ and $\mathrm{H} 4 \mathrm{~A}$. Selected bond lengths $(\AA)$ and angles (deg): Ru1-C20, 1.834(7); Ru1-N1, 2.123(5); Ru1-N2, 2.081(5); Ru1-P1, 2.2738(17); Ru1-H1A, 1.68(8); Ru1-O2, 2.243(4); H4A-O2, 1.867; H5-O2, 1.787; C20-Ru1-N2, 178.3(2); P1-Ru1-N1, 157.83(14); N2-Ru1-O2, 80.60(17).

Reaction of the dearomatized complex $\mathbf{5}$ with ten equivalents of ethylene glycol in a 1:1 mixture of THF $(0.25 \mathrm{~mL})$ and water $(0.25 \mathrm{~mL})$ with no added base was also performed at $115^{\circ} \mathrm{C}$ in an open system, resulting in formation of the glycolic acid adduct 7 as the major product along with minor byproducts (Scheme 4, Figures S53-54, see SI for details). Complex 7 was also obtained by the addition of two equivalents of glycolic acid to complex $5^{\left.5^{25}\right]}$ in THF at room temperature, and its structure was further confirmed by X-ray crystallography (Figure 3 ). The results of the base-free experiment indicate that the first cycle of the reforming reaction to generate the glycolic acid adduct does not require base to promote the $\mathrm{C}-\mathrm{O}$ bond formation. ${ }^{[23]}$ Moreover, upon treatment of sodium 2-hydroxyacetate as a substrate under the optimal conditions no hydrogen evolution occurred, clearly showing that further dehydrogenation of sodium 2-hydroxyacetate did not take place (see SI 6.6 for details).

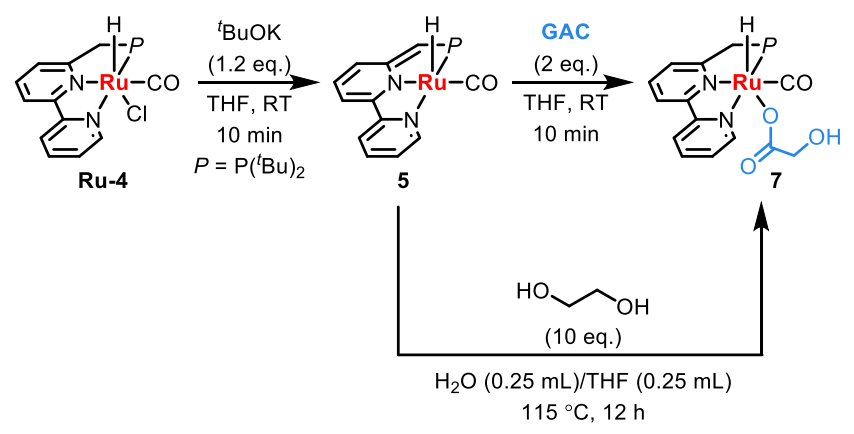

Scheme 4. Dearomatization of complex Ru-4 and reaction with glycolic acid, and reaction with ethylene glycol without base. 


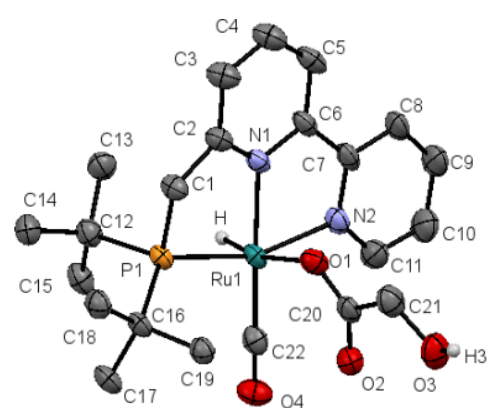

Figure 3. X-ray crystal structure of complex 7. Atoms are presented as therm al ellipsoids at $50 \%$ probability level. Hydrogen atoms are omitted for clarity except the hydride and $\mathrm{H} 3$. Selected bond lengths $(\AA)$ and angles (deg): $\mathrm{Ru}(1)-\mathrm{C}(22)$, 1.845(4); $\mathrm{Ru}(1)-\mathrm{N}(1), 2.086(3) ; \mathrm{Ru}(1)-\mathrm{N}(2), 2.118(3) ; \mathrm{Ru}(1)-\mathrm{O}(1), 2.207(3)$; $\mathrm{Ru}(1)-\mathrm{P}(1), 2.2653(9) ; \mathrm{Ru}(1)-\mathrm{H}, 1.56(5) ; \mathrm{C}(22)-\mathrm{Ru}(1)-\mathrm{N}(1), 178.50(14) ; \mathrm{N}(2)-$ $\mathrm{Ru}(1)-\mathrm{P}(1), 159.96(9) ; \mathrm{N}(1)-\mathrm{Ru}(1)-\mathrm{O}(1), 77.43(11)$.

Following the reaction under standard conditions by ${ }^{31} \mathrm{P}\left\{{ }^{1} \mathrm{H}\right\}$ NMR showed the initial formation of a new species, assigned to be the hydrido-hydroxo complex 8 shown in Scheme 5A (see SI, Figure S55-57, previously also reported for an analogous PNNbased ruthenium complex). ${ }^{[26]}$ Importantly, this shows that even in the presence of excess base, no double deprotonation takes place, excluding a previously proposed anionic reaction surface. ${ }^{[27]}$ This is also in agreement with the base-free reaction outlined earlier (Scheme 4) and might be expected given the protic reaction conditions. In an earlier study, we conjectured that a PNNH-pincer complex might enable challenging reaction schemes by switching between appropriate MLC modes (dearomatization and amido-formation). ${ }^{[24]}$

Employing DFT calculations, we tried to shed light into plausible reaction pathways for this transformation, in particular on three key events: 1) liberation of $\mathrm{H}_{2}, 2$ ) formation of the $\mathrm{C}-\mathrm{O}$ bond, as well as 3) $\mathrm{C}-\mathrm{H}$ bond scission. DFT calculations were performed at the $\omega B$ 97M-V/def2-TZVPP/RIJCOSX/SMD//M06L/def2TZVP/GD3/W0 level of theory (see SI for details). In order to correctly address possible concertedness of transition states, all structures were optimized in solution (THF). ${ }^{[28,29]}$ The trans dihydride 4 is connecting both the $\mathrm{H}_{2}$ liberation step, as well as the $\mathrm{C}-\mathrm{H}$ bond activation step and was thus chosen as a starting point of the mechanistic investigation. Transition states not involving side-arm protons (see SI 8.5) are linked to unreasonably high barriers and were thus excluded. As observed for many other pincer complexes, a proton shuttle is needed for the liberation of $\mathrm{H}_{2}$ from dihydride 4 (Step 1, TS1A, Scheme 5B). Although highly asynchronous, extended IRC-calculations confirm the concertedness of this step (see SI 8.7). Indeed, no stable intermediate is formed after initial protonation of the hydride by the proton shuttle, with subsequent side-arm deprotonation by the conjugated base being linked to the highest energy of this step. ${ }^{[28 b]}$ A more basic proton-shuttle (ethylene glycol) thus gives slightly lower barriers (Scheme 5B, TS1A vs TS1C). As for related PNPcomplexes, the dearomatized species will quickly react with the present protic species, to give either the alkoxo or the hydroxo compounds. The reversibility of the $\mathrm{H}-\mathrm{H}$ and $\mathrm{O}-\mathrm{H}$ activation events is demonstrated experimentally by running the reaction in $\mathrm{D}_{2} \mathrm{O}$ instead of $\mathrm{H}_{2} \mathrm{O}$. $\mathrm{D}_{2}$ liberation was observed by GC-MS, and ${ }^{2} \mathrm{H}-\mathrm{NMR}$ after $12 \mathrm{~h}$ indicates formation of deuterated deuteriodeuterioxo complex 8 (see SI, Figure S58). After a first dehydrogenation event (see $\mathrm{SI}),{ }^{[30]}$ the hydroxo complex 8 is nucleophlic enough to attack the aldehyde intermediate (Step 3, TS3A). This transition state is thus somewhat reminiscent of $\mathrm{C}-\mathrm{N}$ bond cleavage events in Fe-and Mo-catalyzed hydrogenation of amides. ${ }^{[31]}$ The subsequent final $\mathrm{C}-\mathrm{H}$ bond dissociation (Step 4, Scheme $5 \mathrm{~B}$ ) from the geminal diol is found to be step-wise, i.e. the $\mathrm{C}-\mathrm{H}$ bond abstraction involves formally the alkoxide and a cationic Ru-species (TS4B) to regenerate the dihydride 4. It is important to note that no base is needed for the transient generation of the alkoxide in the TOF-determining (TDTS) TS4B. This is in agreement with our experimental results (Scheme 4), where the addition of water and ethylene glycol (but no base) to complex 5 generates the carboxylate 7. As was already postulated in the case of Ru-4, ${ }^{[32]}$ excess base is still necessary in the catalytic system with a twofold role. On the one hand, the overall reaction is endergonic $(+5.5 \mathrm{kcal} / \mathrm{mol})$ and formation of the carboxylate salt would serve as a driving force for the reaction. On the other hand, the formation of carboxylate 10 from 4 and glycolic acid is highly exergonic $(-18.7 \mathrm{kcal} / \mathrm{mol})$ and efficient quenching of glycolic acid by excess base might help to prevent the formation of this off-cycle product, while at the same time increasing the concentration of the hydrido hydroxo complex 8. Several groups highlighted the importance of explicit solvent models for accurate DFT assessment of alcohol dehydrogenation reactions. ${ }^{[28 a, 33]}$ Following the approach of Dub and Gordon, we computed selected key intermediates and transition states in the presence of an explicit water cluster (Scheme 6A). ${ }^{[28 b]}$ Expectedly, the hydrido hydroxo compound $\mathbf{8}$ is highly stabilized by the $\mathrm{H}$ bonding network $(-9.9 \mathrm{kcal} / \mathrm{mol}$ with respect to the dihydride 4 , stabilization of $16.1 \mathrm{kcal} / \mathrm{mol})$. The $\mathrm{H}$-bonding has also a stabilizing effect on the TDTS TS4B ExP $(17.1 \mathrm{kcal} / \mathrm{mol}$, stabilization of $11.8 \mathrm{kcal} / \mathrm{mol}$ ) and the $\mathrm{H}_{2}$ liberating TS1D $\mathrm{DXP}$ $(11.7 \mathrm{kcal} / \mathrm{mol}$, stabilization of $12.4 \mathrm{kcal} / \mathrm{mol})$. As observed in DFT-MD calculations, the $\mathrm{H}$-bond network will decrease the hydricity of the alkoxide species, explaining the lesser degree of stabilization in TS4BExp. Including the explicit water cluster yields an overall energetic span $\delta \mathrm{E}$ of $27.0 \mathrm{kcal} / \mathrm{mol}$, in good agreement with the observed experimental reactivity. Furthermore, the explicit model predicts the hydrido hydroxo compex to be the resting state, which is confirmed experimentally (see $\mathrm{SI}$ ) and predicted for Ru-4 theoretically. ${ }^{[32]}$ The inclusion of explicit solvent molecules is thus critical to correctly identify rate-limiting states on the potential energy surface.

Finally, we were interested in analyzing if the proposed model could account (and explain) the observed difference between PNNH complex Ru-8 and PNN Ru-4 (Scheme 6B). Indeed, the proposed mechanism reproduces qualitatively the slightly higher activity of Ru-8, compared to Ru-4 (TS4B vs. TS4B $\mathbf{B}_{\text {bpy). }}$ ). Importantly, a step-wise deprotonation/hydride transfer sequence can be excluded in the case of Ru-4, highlighting the importance of branching points on the potential energy surface. ${ }^{[34]}$ Whereas in the case of the PNNH compound, deprotonation of the sidearm $\mathrm{NH}$ group is energetically accessible (Scheme 5B, TS4A, $26.1 \mathrm{kcal} / \mathrm{mol}$ ), this is not the case for Ru-4 (TS4A $A_{\text {bpy }}=$ $44.1 \mathrm{kcal} / \mathrm{mol}$, see $\mathrm{SI}$ ). Instead, the $\mathrm{C}-\mathrm{H}$ bond activation via TS4B $_{\text {bpy }}$ (Scheme 6B) might be reached after $\mathrm{C}-\mathrm{O}$ bond formation (see SI 8.4, TS3A bpy $=28.0 \mathrm{kcal} / \mathrm{mol}$ ) via a O $/ \mathrm{H}$ slippage, as proposed previously. ${ }^{[35]}$ Although the ligand framework in the bipyridyl dihydride $\mathbf{1 1}$ is overall sterically less crowded, compared 
to 4 (Scheme 6B, \% $\bigvee_{\text {free }}=41.6$ vs. $\left.\% \bigvee_{\text {free }}=36.1\right)^{[36]}$ and the Rucenters have similar positive NBO-charges of +0.33 , the hydride abstraction in the case of the PNNH-ligand is around $4 \mathrm{kcal} / \mathrm{mol}$ lower in energy compared to the bipyridyl-analogue (Scheme 6B, TS4B $=26.9$ vs TS4B $\left._{\text {bpy }}=30.9 \mathrm{kcal} / \mathrm{mol}\right)$. This difference might be explained by the additional stabilization of the formal alkoxide by the $\mathrm{N}-\mathrm{H}$ moiety in $\mathbf{4}$. Indeed, the $\mathrm{N}-\mathrm{H}$ proton is not only more polarized (NBO-charge of +0.41 vs. +0.27 in 11) but also more accessible in the parent dihydride compound $\mathbf{4}$, as demonstrated by the steric topographic analysis. In the PNNH-ligand, the northwest (NW) quadrant accommodating the $\mathrm{N}-\mathrm{H}$-moiety has an accessible free volume of $\% V_{\text {free }}=22.9$, whereas the quadrants occupied by the ligand backbone $\mathrm{PCH}_{2}$ protons in both ligands are sterically more hindered (e.g. Scheme $6 \mathrm{~B}, \% \mathrm{~V}_{\text {free }}(N E)=10.6$ and $\% V_{\text {free }}(S E)=14.6$ in 11). Indeed, these electronic and steric analyses together indicate that a variety of factors likely govern the difference in activity of the catalysts employed. It might be argued, that the $\mathrm{N}-\mathrm{H}$ bonding is made redundant in the presence of protic reagents/solvents. We nevertheless observe that the stabilization persists in the presence of explicit solvent molecules, with TS4B $_{\text {exp }}$ being around $4 \mathrm{kcal} / \mathrm{mol}$ lower than the corresponding PNN-analogue (TS4B exp $=17.1 \mathrm{kcal} / \mathrm{mol} \mathrm{vs}$. TS4B $_{\text {exp,bpy }}=21.9 \mathrm{kcal} / \mathrm{mol}$, see SI 8.6). Our mechanistic model indeed reproduces qualitatively the trends observed in initial rate experiments run on representative candidates of the PNN and PNNH families (see SI 8.8)

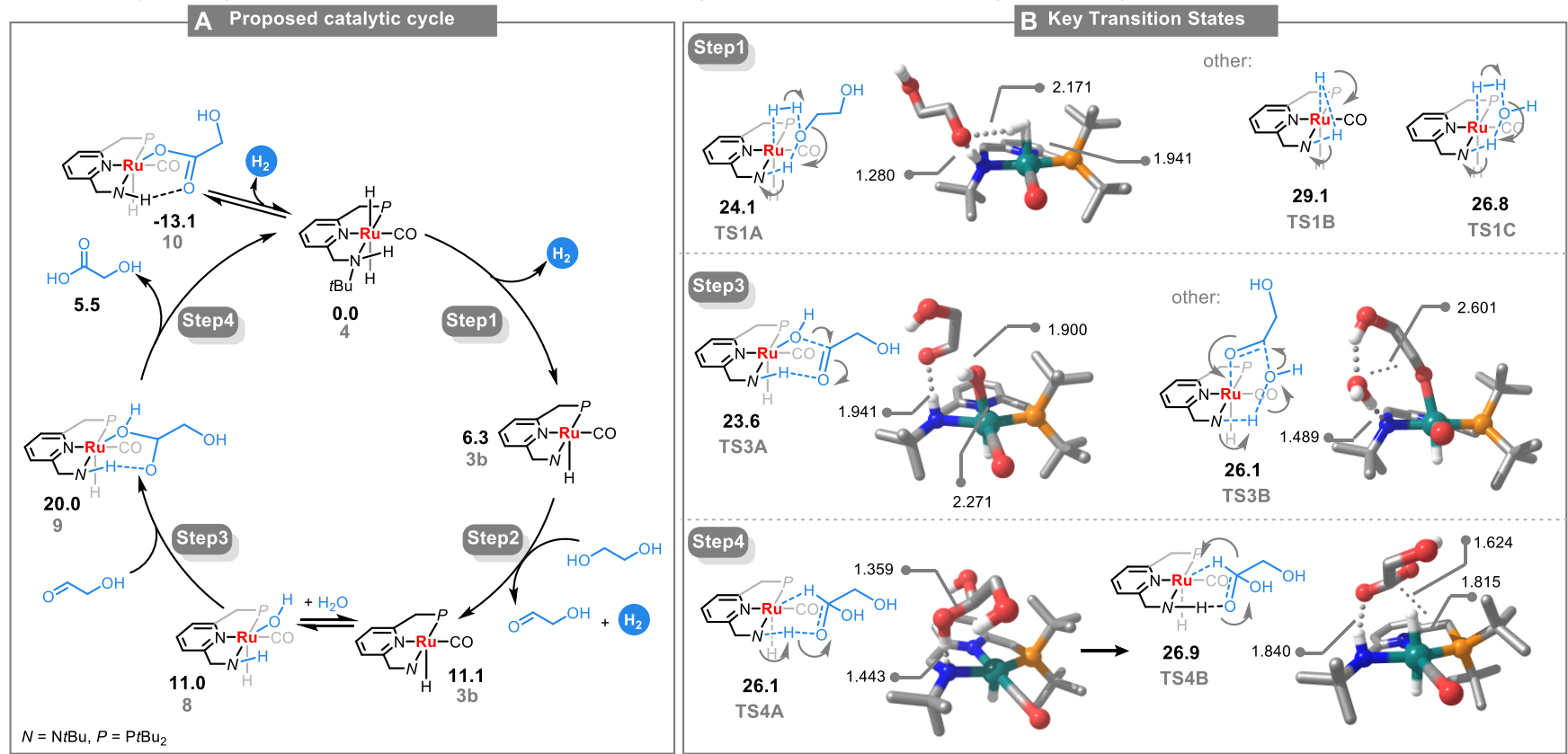

Scheme 5. (A) Proposed catalytic cycle for the reforming of ethylene glycol with water. Values correspond to Gibbs free energies in $\mathrm{kcal}_{\text {.mol }}{ }^{-1}$ with respect to the starting material (0.0). Level of theory: M06-L/def2TZVP/W06/GD3//wBP97M-V/def2TZVPP/RIJCOSX, structures optimized in THF (SMD). (B) Key transition states of the proposed mechanism. Bond lengths in the computed structures in $\AA$.

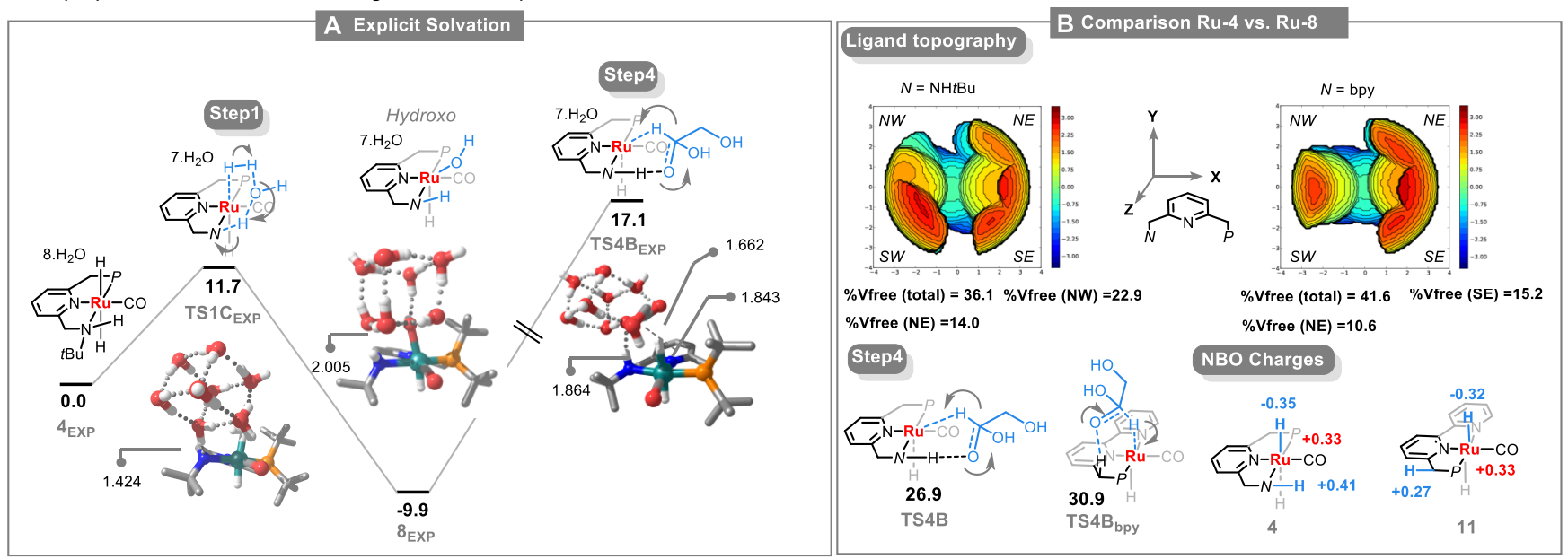

Scheme 6. (A) Selected structures of the potential energy surface in the presence of an explicit water cluster. Values correspond to Gibbs free energies in kcal/mol with respect to the starting material (0.0). Bond lengths in the computed structures in in $\AA$. (B) Comparison of Ru-4 and Ru-8. Steric topographic analysis using SambVca $2.1^{[34]}$ with the ruthenium atom as the center of the sphere (3.5), scaled bond radii (1.17) and 0.1 mesh spacing. 


\section{Conclusion}

In conclusion, we have developed a homogeneous, ruthenium catalyzed, efficient reforming of aqueous ethylene glycol under mild conditions. This reforming process produces glycolic acid (up to $93 \%$ yield) and pure hydrogen gas, without $\mathrm{CO}_{2}$ or $\mathrm{CO}$ emission. The reaction can also be performed on a large scale of $30 \mathrm{mmol}$ of ethylene glycol. Moreover, this reforming process can also proceed well in water with no additional organic solvent. These characteristics make this protocol environmentally friendly, atomeconomical, renewable and sustainable. A plausible reaction mechanism is proposed based on stoichiometric reactions, NMR studies, X-ray crystallography and DFT calculations. The mechanistic studies allow rationalization of the high reactivity of the PNNH ligand framework. It is envisioned that the understanding of the reaction pathways at play might enable future development of new reaction schemes and catalysts.

\section{Acknowledgements}

D.M. holds the Israel Matz Professorial Chair of Organic Chemistry. Y.-Q.Z. acknowledges the Sustainability and Energy Research Initiative (SAERI) of Weizmann Institute of Science for a research fellowship. N.v.W. is supported by the Foreign Postdoctoral Fellowship Program of the Israel Academy of Sciences and Humanities. M.R. acknowledges the Zuckerman STEM Leadership Program for a research fellowship. N.v.W and M.R. thank $\mathrm{Dr}$ Mark Iron (department of chemical research support) for fruitful discussions regarding the DFT calculations. Computations were performed using HPC resources from GENCI-CINES (Grant 2019AP010811227).

Data availabilty: All computed structures and energies can be accessed on the ioChem-BD.org online repository under the following link: https://doi.org/10.19061/iochem-bd-6-66

Keywords: homogeneous catalysis • clean and sustainable • ethylene glycol $\bullet$ glycolic acid $\bullet$ hydrogen

[1] a) B. M. Trost, Science 1991, 254, 1471-1477; b) P. A. Wender, V. A Verma, T. J. Paxton, T. H. Pillow, Acc. Chem. Res. 2008, 41, 40-49; c) T. Newhouse, P. S. Baran, R. W. Hoffmann, Chem. Soc. Rev. 2009, 38, 3010-3021.

[2] Selected reviews: a) T. Ikariya, K. Murata, R. Noyori, Org. Biomol. Chem. 2006, 4, 393-406; b) H. Grützmacher, Angew. Chem. 2008, 120, 18381842; Angew. Chem., Int. Ed. 2008, 47, 1814-1818; c) J. I. van der Vlugt, J. N. H. Reek, Angew. Chem. 2009, 121, 8990-9004; Angew. Chem., Int Ed. 2009, 48, 8832-8846; d) J. I. van der Vlugt, Eur. J. Inorg. Chem. 2012, 363-375; e) J. R. Khusnutdinova, D. Milstein, Angew. Chem. 2015 127, 12406-12445; Angew. Chem., Int. Ed. 2015, 54, 12236-12273.

[3] Selected reviews: a) D. Milstein, Top. Catal. 2010, 53, 915-923; b) C. Gunanathan, D. Milstein, Acc. Chem. Res. 2011, 44, 588-602; c) C Gunanathan, D. Milstein, Science 2013, 341, 1229712-1/11; d) R. H. Crabtree, Chem. Rev. 2017, 117, 9228-9246; e) G. E. Dobereiner, R. H. Crabtree, Chem. Rev. 2010, 110, 681-703; f) S. M. A. H. Siddiki, T. Toyao, K.-i. Shimizu, Green Chem. 2018, 20, 2933-2952; g) F. Raffaella, Curr. Org. Chem. 2018, 22, 1875-1892; h) N. Gorgas, K. Kirchner, Acc.
Chem. Res. 2018, 51, 1558-1569; i) F. Kallmeier, R. Kempe, Angew. Chem. Int. Ed. 2018, 57, 46-60; j) L. Alig, M. Fritz, S. Schneider, Chem. Rev. 2019, 119, 2681-2751; k) K. Sordakis, C. Tang, L. Vogt, H. Junge, P. J. Dyson, M. Beller, Chem. Rev. 2018, 118, 372-433.

[4] J. Kubitschke, H. Lange, H. Strutz in Carboxylic Acids, Aliphatic in Ullmann's Encyclopedia of Industrial Chemistry, Wiley-VCH: Weinheim, 2014

[5] Glycolic Acid Market-Forecasts from 2018 to 2023 https://www.researchandmarkets.com/reports/4542547/glycolic-acidmarket-forecasts-from-2018-to-2023.

[6] S. D. Barnicki, R. T. Hembre, S. N. Falling, M. E. Janka, K. M. Moran US 8703999 B2, 2014

[7] L. Prager, P. Dowideit, H. Langguth, H.-P. Schuchmann, C. von Sonntag, J. Chem. Soc., Perkin Trans. 2001, 2, 1641-1647.

[8] D. Roberts, D. J. Watson, J. Swinney, WO 2018/051115 A1, 2018.

[9] H. Yue, Y. Zhao, X. Ma, J. Gong, Chem. Soc. Rev. 2012, 41, 4218-4244.

[10] A. Wang, T. Zhang, Acc. Chem. Res. 2013, 46, 1377-1386.

[11] R. D. Cortright, R. R. Davda, J. A. Dumesic, Nature 2002, 418, 964-967.

[12] G. W. Huber, J. W. Shabaker, J. A. Dumesic, Science 2003, 300, 20752077.

[13] T. van Haasterecht, T. W. van Deelen, K. P. de Jong, J. H. Bitter, Catal. Sci. Technol. 2014, 4, 2353-2366.

[14] D. Morton, D. J. Cole-Hamilton, J. Chem. Soc., Chem. Commun. 1988 1154-1156.

[15] a) Y. Li, M. Nielsen, B. Li, P. H. Dixneuf, H. Junge, M. Beller, Green Chem 2015, 17, 193-198. For homogeneously reforming aqueous methanol to hydrogen and carbon dioxide, see: b) M. Nielsen, E. Alberico, W. Baumann, H.-J. Drexler, H. Junge, S. Gladiali, M. Beller, Nature 2013, 495, 85-89.

[16] a) T. Zweifel, J.-V. Naubron, H. Grützmacher, Angew. Chem. 2009, 121, 567-571; Angew. Chem. Int. Ed. 2009, 48, 559-563; b) M. Trincado, K Kuhlein, H. Grützmacher, Chem. Eur. J. 2011, 17, 11905-11913.

[17] Y. Zhan, W. Hou, G. Li, Y. Shen, Y. Zhang, Y. Tang, ACS Sustainable Chem. Eng. 2019, 7, 17559-17564.

[18] J. Wu, L. Shen, Z.-N. Chen, Q. Zheng, X. Xu, T. Tu, Angew. Chem. 2020 132, 10507-10511; Angew. Chem. Int. Ed. 2020, 59, 10421-10425.

[19] J. Zhang, G. Leitus, Y. Ben-David, D. Milstein, J. Am. Chem. Soc. 2005 127, 10840-10841.

[20] C. Gunanathan, Y. Ben-David, D. Milstein, Science 2007, 317, 790-792

[21] a) E. Balaraman, E. Khaskin, G. Leitus, D. Milstein, Nat. Chem. 2013, 5 122-125; b) P. Hu, Y. Ben-David, D. Milstein, J. Am. Chem. Soc. 2016, $19,6143-6146$

[22] Y.-Q. Zou, N. von Wolff, A. Anaby, Y. Xie, D. Milstein, Nat. Catal. 2019 2, 415-422.

[23] It is important to note that the reaction mixture is biphasic. Excess base is used to quench any formed acidic product, both to drive the reaction to completion and to prevent catalyst degradation or deactivation. Indeed, DFT calculations predict that the reaction is endergonic and hence formation of the glycolate salt from glycolic acid might be an additional driving force of the reaction (besides irreversible $\mathrm{H}_{2}$ release).

[24] E. Fogler, J. A. Garg, P. Hu, G. Leitus, L. J. W. Shimon, D. Milstein, Chem. Eur. J. 2014, 20, 15727-15731.

[25] D. Milstein, E. Balaraman, C. Gunanathan, B. Gnanaprakasam, J. Zhang, US 2013/0281664 A1, 2013.

[26] S. W. Kohl, L. Weiner, L. Schwartsburd, L. Konstantinovski, L. J. W. Shimon, Y. Ben-David, M. A. Iron, D. Milstein, Science 2009, 324, 7477.

[27] C. Hou, J. Jiang, Y. Li, C. Zhao, Z. Ke, ACS Catal. 2017, 7, 786-795.

[28] a) D. G. Gusev, Organometallics 2020, 39, 258-270; b) P.A. Dub, J. C. Gordon, ACS Catal. 2017, 7, 6635-6655.

[29] Note however, that the experimentally observed site of deprotonation is only correctly predicted following the original procedure for parametrization of Cramer and Truhlar, i.e. optimizing in the gas phase: a) R. F. Ribeiro, A. V. Marenich, C. J. Cramer, D. G. Truhlar, J. Phys. Chem. B, 2011, 115, 14556-14562; b) Marenich, C. J. Cramer, D. G. Truhlar, J. Phys. Chem. B, 2009, 113, 6378-6396. No standard state 
correction for $1 \mathrm{~atm}$ to $1 \mathrm{M}$ was applied, nor $\mathrm{H}_{2}$ solubility taken into account. These corrections can be applied subsequently.

[30] Step 2 resembles Step 4, but is significantly lower in energy.

[31] a) L. A. Suàrez, Z. Culakova, D. Balcells, W. H. Bernskoetter, O. Eisenstein, K. I. Goldberg, N. Hazari, M. Tilset, A. Nova, ACS Catal. 2018 8, 8751-8762 b) T. Leischner, L. A. Suarez, A. Spannenberg, K. Junge, A. Nova, M. Beller Chem. Sci. 2019,10, 10566-10576

[32] H. Li, M. B. Hall, J. Am. Chem. Soc. 2014, 136, 383-395

[33] a) V. Sinha, N. Govindarajan, B. de Bruin, E. J. Meijer ACS Catal. 2018, 8, 6908-6913 b) N. Govindarajan, V. Sinha, M. Trincado, H. Grützmacher, E. J. Meijer, B. de Bruin, ChemCatChem 2020, 12, 2610.

[34] P.A. Dub, J. C. Gordon, Dalton Trans., 2016, 45, 6756-6781.

[35] F. Hasanayn, L. M. Al-Assi, R. N. Moussawi, B. S. Omar, Inorg. Chem. 2016, 55, 7886-7902.

[36] L. Falivene, Z. Cao, A. Petta, L. Serra, A. Poater, R. Oliva, V. Scarano, L. Cavallo, Nat. Chem. 2019, 11, 872-879 


\section{Entry for the Table of Contents}

\section{RESEARCH ARTICLE}

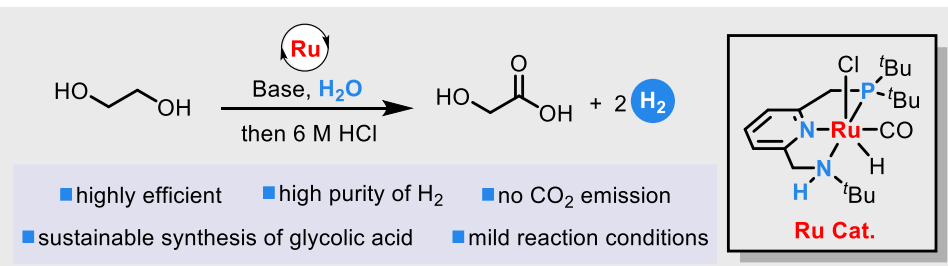

A homogeneous ruthenium-catalyzed reforming of aqueous ethylene glycol to glycolic acid and hydrogen is described. A plausible reaction mechanism, involving metal ligand cooperation is proposed and supported by stoichiometric reactions, NMR studies, X-ray crystallography and computational studies.
Y.-Q. Zou, N. von Wolff, M. Rauch, M. Feller, Q.-Q. Zhou, A. Anaby, Y. DiskinPosner, L. J. W. Shimon, L. Avram, Y. Ben-David, and D. Milstein*

Page No. - Page No.

Homogeneous Reforming of Aqueous Ethylene Glycol to Glycolic Acid and Pure Hydrogen Catalyzed by PincerRuthenium Complexes Capable of Metal-Ligand Cooperation 